Pesq. Vet. Bras. 37(11):1352-1356, novembro 2017

DOI: $10.1590 / \mathrm{S} 0100-736 \mathrm{X} 2017001100026$

\title{
Variação nos tipos de irrigação do nodo sinoatrial em cães de diferentes raças $^{1}$
}

\author{
Phelipe O. Favaron ${ }^{2 *}$, Jéssica Borghesi², Rafael G. Agopian³ ${ }^{3}$ Maria Angelica \\ Miglino $^{2}$ e Vicente Borelli ${ }^{4}$
}

\begin{abstract}
Favaron P.O., Borghesi J., Agopian R.G., Miglino M.A. \& Borelli V. 2017. [Variation in irrigation of the sinoatrial node in dogs of different breeds.] Variação nos tipos de irrigação do nodo sinoatrial em cães de diferentes raças. Pesquisa Veterinária Brasileira 37(11):1352-1356. Setor de Anatomia dos Domésticos e Silvestres, Faculdade de Medicina Veterinária e Zootecnia, Universidade de São Paulo, Rua Professor Orlando Marques de Paiva 87, Butantã, São Paulo, SP 05508-270, Brazil. E-mail: *E-mail: phelipe.favaron@yahoo.com.br

Considering the anatomical and electrophysiological heterogeneity of the sinoatrial node for generation and propagation of the action potential, as well as the particularities relating to the origin of blood nutrition, this study aimed to analyze the behavior of arteries involved in irrigation of the sinoatrial node in dogs performing a descriptive and comparative analysis between different breeds, with emphasis in the origin, pathway and branching of vessels, as well as the presence of anastomoses. Totally, 240 hearts were fixed in solution of formalin $10 \%$ and subjected to Spalteholz diaphanization. The vascularization of the sinoatrial node occurs by the right circumflex branch or left circumflex branch, showing several particularities according to the breed. Thus, predominantly in the studied breeds, the blood supply of the sinoatrial node depends exclusively (63.6\%) or from anastomosis of the right coronary artery. There is also participation of right atrial proximal branch and right atrial intermediary branch. Less often $(15.4 \%)$ the blood supply occurs exclusively from the left atrial proximal branch, which is a branch of the circumflex branch of the left coronary artery. In summary, our results related to the origin of the sinoatrial node blood nutrition and the branching of vessels involved on that represents a fundamental knowledge for the development and improvement of surgery in dogs, as well as for pathology and experimental research.
\end{abstract}

INDEX TERMS: Irrigation, sinoatrial node, dogs, coronary, arterial vascularization, anastomosis, animal models.

RESUMO.- Considerando a heterogeneidade anatômica e eletrofisiológica do nodo sinoatrial para a geração e propagação do potencial de ação, bem como as particularidades relacionadas a origem da sua irrigação sanguínea, este trabalho teve como objetivo analisar o comportamento das

\footnotetext{
${ }^{1}$ Recebido em 8 de agosto de 2016.

Aceito para publicação em 14 de fevereiro de 2017.

${ }^{2}$ Setor de Anatomia dos Domésticos e Silvestres, Faculdade de Medicina Veterinária e Zootecnia, Universidade de São Paulo, Rua Professor Orlando Marques de Paiva 87, Butantã, São Paulo, SP, 05508-270, Brasil. E-mails: jehborghesi@hotmail.com, miglino@usp.br; *Autor para correspondência: phelipe.favaron@yahoo.com.br

${ }^{3}$ Departamento de Morfologia, Universidade Paulista (Unip), Rua Professor Enéas de Siqueira Neto 340, Jardim das Imbuias, São Paulo, SP 04829-300, Brasil. E-mail: rafael.agopian@gmail.com

${ }^{4}$ Departamento de Anatomia, Unip, Rua Doutor Bacelar 1212, Vila Clementino, São Paulo, SP 04026-002, Brasil. E-mail: v.borelli@terra.com.br
}

artérias envolvidas na irrigação do nodo sinoatrial em cães realizando uma análise descritiva e comparativa entre diferentes raças estudadas, detalhando a origem, o percurso e a ramescência dos vasos, assim como a eventual ocorrência de anastomoses. Ao todo analisamos resultados obtidos em 240 corações, os quais foram fixados em solução de formalina $10 \%$ e submetidos a diafanização de Spalteholz. A irrigação deste ocorre mediante colaterais oriundos do ramo circunflexo direito ou ramo circunflexo esquerdo, mostrando particularidades diferentes para cada raça. Assim, predominantemente, nas raças ora estudadas a irrigação arterial do nodo sinoatrial depende exclusivamente $(63,6 \%)$, ou de anastomoses de colaterais da artéria coronária direita, havendo também participação dos ramo proximal atrial direito e intermédio atrial direito. Menos frequentemente $(15,4 \%)$ o suprimento sanguíneo ocorre exclusivamente por conta do ramo proximal atrial esquerdo, oriundo do 
ramo circunflexo da artéria coronária esquerda. Os dados aqui apresentados sobre a origem da irrigação sanguínea do nodo sinoatrial e a ramescência dos vasos envolvidos nesta tarefa representam conhecimento fundamental para o desenvolvimento da clínica-cirúrgica em cães, da patologia e trabalhos de natureza experimental.

TERMOS DE INDEXAÇÃO: Irrigação, nodo sinoatrial, cães, coronárias, vascularização arterial, anastomose, modelos animais.

\section{INTRODUÇÃO}

Diante da importância funcional do nodo sinoatrial para o automatismo cardíaco, inúmeros pesquisadores têm se interessado pelo entendimento dos aspectos morfofuncionais que determinam não apenas a frequência cardíaca, mas também seus batimentos, regeneração celular e transplante (Lobo et al. 2012, Glukhov et al. 2013, Zhang et al. 2013). O nodo sinoatrial é uma estrutura heterogênea tanto do ponto de vista anatômico quanto eletrofisiológico, e expressa um conjunto único de canais iônicos necessários para a geração e propagação do potencial de ação (Monfredi et al. 2010).

Em cães, depois da descoberta por Keith \& Flack (1907), inúmeros pesquisadores estudaram os aspectos histológicos, localização, topografia e fisiologia do nodo sinoatrial (James 1962, James 1966, Lobo et al. 2012). Estas informações tornaram-se indispensáveis para o estabelecimento de protocolos aplicados na prática cirúrgica (Zhang et al. 2011, 2013, Glukhov et al. 2013). Os diferentes experimentos realizados por Pereira et al. (1972) sobre infarto atrial experimental em cães sem raça definida (SRD), em especial os realizados mediante ligadura cirúrgica das artérias responsáveis pelo suprimento sanguíneo do tecido nodal, tiveram grande importância nesse contexto.

Um ponto fundamental nessa questão está relacionado com a origem da irrigação sanguínea do nodo sinoatrial, sobre o qual é sabido existir tipos diferentes para as várias espécies e raças, como demonstrado previamente para cães (Andretto 1972, Fernandes Filho et al. 1975, Amaral 1982, Randi 1988, Biasi et al. 2013).

Nesse sentido, o objetivo deste trabalho foi estudar o comportamento das artérias envolvidas na irrigação do nodo sinoatrial em diferentes raças de cães realizando uma análise comparativa, em particular, no que tange a origem, o percurso e a ramescência dos referidos vasos, assim como a eventual ocorrência de anastomoses entre eles, fornecendo dessa maneira, subsídios indispensáveis para o desenvolvimento da cirurgia experimental e comparativa.

\section{MATERIAL E MÉTODOS}

Ao todo, foram analisados 240 corações de cães, sendo destes: 30 da raça Boxer (15 machos e 15 fêmeas), 27 Poodle (19 machos e 8 fêmeas), 30 Doberman (22 machos e 8 fêmeas), 50 Pastor alemão (38 machos e 12 fêmeas), 40 Pequinês (22 machos e 18 fêmeas), e 63 SRD ( 40 machos e 23 fêmeas) com diferentes e desconhecidas idades. Os animais foram oriundos do Hospital Veterinário da Faculdade de Medicina Veterinária e Zootecnia da Universidade de São Paulo, São Paulo, Brasil. 0 projeto foi avaliado e aprovado pelo Comitê de Ética em Pesquisa no Uso de Animais da Universidade de Santo Amaro, São Paulo (Parecer n.10/2016).
Inicialmente os corações foram isolados e os átrios e ventrículos lavados em água corrente. As artérias coronárias foram injetadas com solução aquosa de gelatina a 10\% (p/v) corada com cinábrio (natural mercury sulfide CARLO ERBA S/A). Em seguida, os corações foram fixados em solução de formalina $10 \%$ por 48 horas e submetidos ao processo de diafanização de Spalteholz. Desenhos esquemáticos foram realizados para documentação. Além disso, a fim de verificar a ocorrência de anastomoses arteriais, com participação dos vasos responsáveis pela irrigação da região ocupada pelo nodo sinoatrial em cães, um estudo detalhado das mesmas foi realizado nos corações das raças doberman, pastor alemão e pequinês. Foi empregada a nomenclatura baseada na Veterinary Anatomical Nomenclature (2012). Os dados foram analisados estatisticamente utilizando o Test $\mathrm{X}^{2}$.

\section{RESULTADOS}

\section{Irrigação arterial da região ocupada pelo nodo sinoatrial}

A irrigação do nodo sinoatrial mostrou variações importantes de acordo com as raças analisadas. Nestas, a irrigação arterial do território tomado pelo nodo sinoatrial dependia de vasos oriundos da artéria coronária direita, isto é do ramo circunflexo direito ou ramo circunflexo esquerdo, este oriundo da artéria coronária esquerda ou tinha a contribuição de vasos oriundos de ambas as coronárias (Quadro 1).

Predominantemente, em todas as raças analisadas, a irrigação arterial do território ocupado pelo nodo sinoatrial dependia exclusivamente do ramo distal atrial direito, visto a nascer do ramo circunflexo direito (Fig.1A,B). Esse tipo ocorreu em $73,3 \%( \pm 8,1)$ no boxer, $55,5 \%( \pm 9,2)$ no poodle, $90 \%( \pm 8,4)$ no doberman, $42 \%( \pm 7,0)$ no pastor alemão, $65 \%( \pm 7,5)$ no pequinês e $55,5 \%( \pm 6,26)$ em SRD. Nestes casos, logo após a origem, o ramo distal atrial direito ganha à superfície do átrio direito, seguindo, com trajeto ascendente em direção à desembocadura da veia cava cranial, para, como tronco ou mediante colateral percorrer o sulco terminal em toda sua extensão, ou apenas parcialmente. No trajeto descrito, o ramo distal atrial direito cedia ainda colaterais à face atrial do átrio direito, à seção final da veia cava caudal e ao segmento terminal da veia cava cranial.

0 ramo proximal atrial direito (Fig.1C,D) foi identificado 2 vezes no boxer e uma única vez em poodle e em SRD, numa frequência de 6,6\%,3,7\% e 1,58\%, respectivamente. Este vaso, após sua origem, contorna parcialmente a aorta próximo de sua emergência, passa dela desviando-se visto a seguir, como tronco, à face auricular do átrio direito, com trajeto ascendente, para, rumo a desembocadura da veia cava cranial, atingir e percorrer diretamente o ângulo diedro cavo atrial e o sulco terminal em toda sua extensão. 0 ramo proximal atrial direito neste trajeto cedia ainda contribuição que volteia parcialmente a aorta, junto à origem, bem como colaterais à face auricular e atrial do átrio direito e a secção final da veia cava cranial. 0 ramo intermédio atrial direito (Fig.1E,F) por sua vez, ocorreu apenas uma única vez nutrindo o tecido nodal do boxer $(3,3 \%)$, doberman $(3,3 \%)$ e pastor alemão $(2 \%)$ e 8 vezes na raça pequinês (20\%), não sendo observado no poodle e SRD. 0 ramo intermédio atrial direito ao deixar o ramo circunflexo direito passava a percorrer com trajeto ascendente, a face atrial do átrio direito, cedendo-lhes colaterais, em busca da veia cava cranial, para seguir por toda a extensão do sulco terminal e atingir o ângulo diedro cavo atrial. 
Em 13,3\% $( \pm 6,2)$ dos corações de boxer, 29,63\% \pm $14,3)$ no poodle, $18,0 \%( \pm 5,4)$ no pastor alemão, $5 \%( \pm$ $3,4)$ no pequinês e $11,11 \%( \pm 3,96)$ em SRD, o suprimento sanguíneo do nodo sinoatrial ficava na dependência exclusiva do ramo proximal atrial esquerdo (Fig.1G,H), oriundo do segmento inicial do ramo circunflexo da artéria coronária esquerda. Este vaso acompanhava, após a origem, a superfície convexa dos átrios, com destino à desembocadura da veia cava cranial, para, à custa de vaso resultante de sua divisão, como tronco, ou mediante colateral, percorrer o ângulo diedro cavo atrial e toda a extensão do sulco terminal. 0 ramo proximal atrial esquerdo, no seu percurso cedia ainda contribuições às faces auricular e atrial da aurícula e átrio esquerdo, à região das veias pulmonares, ao septo interatrial, quando lá não chegava diretamente, a superfície atrial do átrio direito, e ao trato terminal da veia cava cranial. Uma única vez no pastor alemão houve presença exclusiva do ramo intermédio atrial direito na irrigação do nodo sinoatrial (Fig.1I,J). Por outro lado, somente no doberman não foi identificada a participação do ramo proximal atrial esquerdo na irrigação do aludido nodo.

No caso em que a irrigação da área tomada pelo nodo sinoatrial possuía uma dependência parcial de ramificações oriundas dos ramos circunflexos direito e esquerdo, com maior frequência houve participação de ambos o ramo proximal atrial esquerdo e ramo distal atrial direito (Fig.1K,L),

Quadro 1. Comparação entre a irrigação do nodo sinoatrial de diferentes raças de cães

\begin{tabular}{|c|c|c|c|c|c|c|c|}
\hline \multirow{2}{*}{\multicolumn{2}{|c|}{ Vasos }} & \multicolumn{6}{|c|}{ Frequência \% } \\
\hline & & $\begin{array}{l}\text { Boxer } n=30 \\
(15 \mathrm{M} \mathrm{e} 15 \mathrm{~F})\end{array}$ & $\begin{array}{c}\text { Poodle } n=27 \\
(19 M \text { e } 8 F)\end{array}$ & $\begin{array}{c}\text { Doberman } n=30 \\
(22 \mathrm{M} \text { e } 8 \mathrm{~F})\end{array}$ & $\begin{array}{c}\text { Pastor alemão } n=50 \\
(38 \mathrm{M} \text { e } 12 \mathrm{~F})\end{array}$ & $\begin{array}{c}\text { Pequinês } n=40 \\
(22 \mathrm{M} \text { e } 18 \mathrm{~F})\end{array}$ & $\begin{array}{c}\text { SRD n }=63 \\
(40 \mathrm{M} \mathrm{e} 23 \mathrm{~F})\end{array}$ \\
\hline \multirow{3}{*}{$\begin{array}{l}\text { Ramo } \\
\text { circunflexo } \\
\text { direito }\end{array}$} & $\begin{array}{l}\text { Ramo distal atrial } \\
\text { direito (Fig.1A,B) }\end{array}$ & $\begin{array}{c}22 \text { vezes } \\
(73,3 \% \pm 8,1)\end{array}$ & $\begin{array}{c}15 \text { vezes } \\
(55,55 \% \pm 9.2)\end{array}$ & $\begin{array}{c}27 \text { vezes } \\
(90 \% \pm 8,4)\end{array}$ & $\begin{array}{c}21 \text { vezes } \\
(42,0 \% \pm 7,0)\end{array}$ & $\begin{array}{c}26 \text { vezes } \\
(65,0 \% \pm 7,5)\end{array}$ & $\begin{array}{c}35 \text { vezes } \\
(55,55 \% \pm 6,26)\end{array}$ \\
\hline & $\begin{array}{l}\text { Ramo proximal atrial } \\
\text { direito (Fig.1C,D) }\end{array}$ & $\begin{array}{l}\text { Duas vezes } \\
(6,6 \% \pm 4,5)\end{array}$ & $\begin{array}{c}\text { Uma vez } \\
(3,70 \% \pm 2,6)\end{array}$ & - & - & - & $\begin{array}{c}\text { Uma vez } \\
(1,58 \% \pm 1,57)\end{array}$ \\
\hline & $\begin{array}{l}\text { Ramo intermédio atrial } \\
\text { direito (Fig.1E,F) }\end{array}$ & $\begin{array}{c}\text { Uma vez } \\
(3,3 \% \pm 3,3)\end{array}$ & - & $\begin{array}{c}\text { Uma vez } \\
(3,3 \% \pm 2,1)\end{array}$ & $\begin{array}{c}\text { Uma vez } \\
(2,0 \% \pm 2,0)\end{array}$ & $\begin{array}{c}8 \text { vezes } \\
(20,0 \% \pm 6,3)\end{array}$ & - \\
\hline \multirow{2}{*}{$\begin{array}{l}\text { Ramo } \\
\text { circunflexo } \\
\text { esquerdo }\end{array}$} & $\begin{array}{l}\text { Ramo proximal atrial } \\
\text { esquerdo (Fig.1G,H) }\end{array}$ & $\begin{array}{c}4 \text { vezes } \\
(13,3 \% \pm 6,2)\end{array}$ & $\begin{array}{c}8 \text { vezes } \\
(29,63 \% \pm 14,3)\end{array}$ & - & $\begin{array}{c}9 \text { vezes } \\
(18,0 \% \pm 5,4)\end{array}$ & $\begin{array}{l}\text { Duas vezes } \\
(5,0 \% \pm 3,4)\end{array}$ & $\begin{array}{c}7 \text { vezes } \\
(11,11 \% \pm 3,96)\end{array}$ \\
\hline & $\begin{array}{l}\text { Ramo intermédio atrial } \\
\text { esquerdo (Fig.1I,J) }\end{array}$ & & - & & $\begin{array}{c}\text { Uma vez } \\
(2.0 \% \pm 2.0)\end{array}$ & & \\
\hline \multirow[t]{3}{*}{ Ambos } & $\begin{array}{l}\text { Ramo proximal atrial } \\
\text { esquerdo e ramo distal } \\
\text { atrial direito (Fig.2A,B) }\end{array}$ & $\begin{array}{c}\text { Uma vez } \\
(3,3 \% \pm 3,3)\end{array}$ & $\begin{array}{c}3 \text { vezes } \\
(11,11 \% \pm 3,9)\end{array}$ & $\begin{array}{l}\text { Duas vezes } \\
(6,7 \% \pm 3,2)\end{array}$ & $\begin{array}{c}13 \text { vezes } \\
(26,0 \% \pm 6,2)\end{array}$ & $\begin{array}{c}3 \text { vezes } \\
(7,5 \% \pm 3,9)\end{array}$ & $\begin{array}{c}20 \text { vezes } \\
(31,74 \% \pm 5,86)\end{array}$ \\
\hline & $\begin{array}{l}\text { Ramo intermédio atrial } \\
\text { direito e ramo proximal } \\
\text { atrial esquerdo (Fig.2C,D) }\end{array}$ & - & - & - & - & $\begin{array}{c}\text { Uma vez } \\
(2,5 \% \pm 2,5)\end{array}$ & - \\
\hline & $\begin{array}{l}\text { Ramo intermédio atrial } \\
\text { direito e ramo proximal } \\
\text { atrial direito (Fig.2E,F) }\end{array}$ & - & - & - & $\begin{array}{c}4 \text { vezes } \\
(8,0 \% \pm 3,8)\end{array}$ & - & - \\
\hline
\end{tabular}
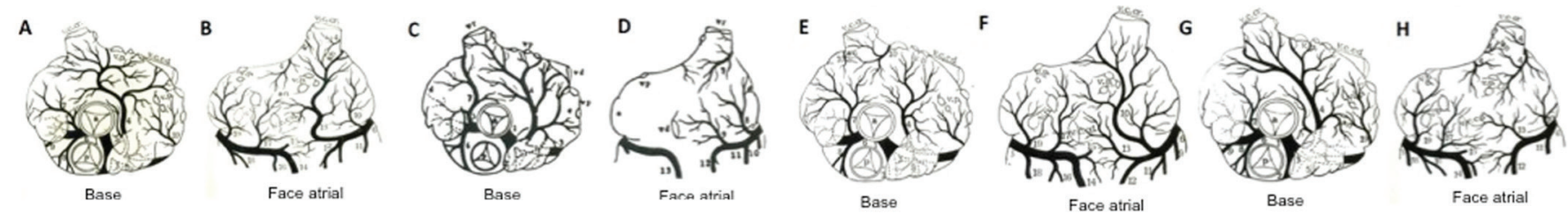

I


K


Fig.1. Tipos de irrigação do nodo sinoatrial em cães. (A-F) Contribuição de ramos oriundos somente do ramo circunflexo direito. Em [AB] ramo distal atrial direito. Em (C-D) ramo proximal atrial direito. Em (E-F) ramo intermédio atrial direito. Em (G-J) contribuição de ramos oriundos somente do ramo circunflexo esquerdo. Em (G-H) ramo proximal atrial esquerdo. Em (I-J) ramo intermédio atrial esquerdo. (K-P) Tipos de irrigação do nodo sinoatrial em cães com contribuição de vasos oriundos dos ramos circunflexo direito e ramo circunflexo esquerdo. Em (K e L) ramo proximal atrial esquerdo e ramo distal atrial direito. Em (M e $\mathbf{N}$ ) ramo intermédio atrial direito e ramo proximal atrial esquerdo. Em (0 e P) ramo intermédio atrial direito e ramo proximal atrial direito. Notar: óstio aórtico (a), óstio pulmonar (b), artéria pulmonar (p), veia cava cranial (v.c.cr), veia cava caudal (v.c.cd), veia pulmonar (v.p), anastomose (an); artéria coronária esquerda (1), ramo interventricular paraconal (2), ramo circunflexo esquerdo (3), ramo proximal atrial esquerdo (4), ramo proximal ventricular esquerdo (5), artéria coronária direita (6), ramo proximal atrial direito (7), artéria adiposa (8), ramo proximal ventricular direito (9), ramo intermédio atrial direito (10), ramo marginal agudo (11), ramo distal ventricular direito (12), ramo distal atrial direito (13), ramo descendente subsinuoso (14), ramo ventricular direito (15), ramo distal ventricular esquerdo (16), ramo distal atrial esquerdo (17), ramo marginal obtuso (18) e ramo intermédio atrial esquerdo (19). 
ocorrendo uma vez $(3,3 \% \pm 3,3)$ no boxer, 3 vezes $(11,11 \%$ $\pm 3,9)$ em poodle, 2 vezes $(6,7 \% \pm 3,2)$ em doberman, 13 vezes $(26 \% \pm 6,2)$ em pastor alemão, 3 vezes $(7,5 \% \pm 3,9)$ em pequinês e 20 vezes $(31,74 \% \pm 5,9)$ em SRD. Nestas ocorrências, o ramo proximal atrial esquerdo nascia do trecho inicial do ramo circunflexo da artéria coronária esquerda, acompanhava a curvatura da superfície côncava dos átrios, ora em plena espessura dos feixes musculares atriais, ora logo abaixo do epicárdio para, depois de alcançar o ângulo diedro cavo atrial, atingir diretamente os terços dorsal e médio do sulco terminal. 0 ramo proximal atrial esquerdo, no seu trajeto, fornecia colaterais às superfícies auricular e atrial do átrio esquerdo e correspondente aurícula, à região das veias pulmonares, ao septo interatrial, às faces auricular e atrial do átrio direito e ao segmento final da veia cava cranial.

Com frequência menor, ocorreu apenas uma vez $(2,5 \%$ $\pm 2,5$ ) no pequinês a participação de ambos, os ramo intermédio atrial direito e ramo proximal atrial esquerdo (Fig.1M,N). Além disso, no pastor alemão ocorreram em 4 casos $(8 \% \pm 3,8)$ a presença dos ramo intermédio atrial direito e ramo proximal atrial direito (Fig.10,P), e uma única vez $(2 \% \pm 2,0)$ à participação concomitante dos ramo proximal atrial direito e distal atrial direito.

Diferenças relacionadas ao sexo, quanto à participação exclusiva de colaterais do ramo circunflexo direito ou do ramo circunflexo da artéria coronária direita na irrigação do nodo sinoatrial, e de vasos oriundos somente do ramo circunflexo da artéria coronária esquerda, mostraram-se estatisticamente significantes, ao nível de 5\%, somente no pastor alemão, bem como à intervenção dos ramos procedentes de ambas as artérias coronárias.

\section{Anastomoses}

No que tange há ocorrência de anastomoses arteriais, com participação dos vasos responsáveis pela irrigação da região ocupada pelo nodo sinoatrial, o pastor alemão foi o que apresentou maior diversidade (12 tipos). Uma distribuição comparativa das anastomoses encontradas pode ser observada na Figura 2.

Com maior frequência, nas 3 raças, foram identificadas anastomoses entre os ramo proximal atrial esquerdo e ramo distal atrial direito, ocorrendo 9 vezes $(56,25 \%), 22$ vezes $(35,48 \%)$ e 9 vezes $(69,23 \%)$, em doberman, pastor alemão e pequinês, respectivamente. Nas 3 raças ocorreram também anastomoses entre os ramo proximal atrial direito e ramo distal atrial direito, sendo uma única vez nas raças doberman $(6,25 \%)$ e pequinês $(7,69 \%)$ e 2 vezes $(3,22 \%)$ no pastor alemão. A raça doberman apresentou ainda anastomoses entre os ramo proximal atrial direito e ramo proximal atrial esquerdo ( 2 vezes, $12,5 \%$ ), entre os ramo distal atrial esquerdo e ramo distal atrial direito (3 vezes, $18,75 \%$ ) e entre os ramo distal atrial direito e ramo ventricular direito (1 vez, 6,25\%). Por outro lado, na raça pequinês identificou-se anastomoses entre os colaterais do ramo proximal atrial esquerdo ( $1 \mathrm{vez}, 7,7 \%)$ e entre os ramo intermédio atrial direito e proximal atrial esquerdo ( 2 vezes, 15,38\%). No pastor alemão anastomoses foram vistas entre os ramo proximal atrial esquerdo e distal atrial esquerdo ( 5 vezes, $8,06 \%$ ), ramo proximal atrial esquerdo



Fig.2. Participação de anastomoses na irrigação arterial do nodo sinoatrial em cães das raças doberman, pastor alemão e pequinês. Ramo proximal atrial esquerdo e distal atrial direito (1), ramo proximal atrial esquerdo e distal atrial esquerdo (2), ramo proximal atrial esquerdo e intermédio atrial esquerdo (3), ramo proximal atrial esquerdo e ventricular direito (4), ramo proximal atrial esquerdo (5), ramo proximal atrial direito e distal atrial direito (6), ramo proximal atrial direito e atrial esquerdo (7), ramo proximal atrial direito e intermédio atrial direito (8), ramo proximal atrial direito e intermédio atrial esquerdo (9), ramo distal atrial esquerdo e distal atrial direito (10), ramo distal atrial direito e ramo ventricular direito (11), ramo distal atrial direito e intermédio atrial esquerdo (12), ramo intermédio atrial esquerdo (13), ramo intermédio atrial direito e proximal atrial esquerdo (14) e ramo intermédio atrial direito (15).

e intermédio atrial esquerdo ( 9 vezes, $14,52 \%$ ), ramo proximal atrial esquerdo e ventricular direito (1 vez, 1,61\%), colaterais do ramo proximal atrial esquerdo (2 vezes, $3,22 \%)$, ramo proximal atrial direito e intermédio atrial direito (5 vezes, $8,06 \%$ ), ramo proximal atrial direito e intermédio atrial esquerdo ( $1 \mathrm{vez}, 1,61 \%)$, ramo distal atrial esquerdo e distal atrial direito (10 vezes, 16,13\%), ramo distal atrial direito e intermédio atrial esquerdo (3 vezes, $4,84 \%$ ) e colaterais dos ramos intermédio atrial esquerdo (1 vez, 1,61\%) e do intermédio atrial direito ( 1 vez, 1,61\%).

\section{DISCUSSÃO}

O conhecimento sobre a natureza do funcionamento do coração dos vertebrados sempre despertou o interesse dos pesquisadores, especialmente, do ponto de vista eletrofisiológico, onde o nodo sinoatrial é a estrutura responsável pela geração e propagação do potencial de ação (Monfredi et al. 2010). Especialmente em cães, há mais de um século o nodo sinoatrial tem sido motivo de pesquisas, inicialmente com trabalhos sobre sua topografia e aspectos histológicos (Keith \& Flack 1907, Rothberger \& Scherf 1926), e mais recentemente sobre a caracterização da sua irrigação sanguínea (Fernandes Filho et al. 1975, Amaral 1982, Biasi et al. 2013). Estas pesquisas mostraram existir tipos diversos para as diferentes raças (Andretto 1972, Fernandes Filho et al. 1975, Amaral 1982, Randi 1988), levando-nos a desenvolver uma análise comparativa dos padrões de irrigação sanguínea do nodo sinoatrial existentes em cães.

Predominantemente, em todas as raças de cães analisadas (boxer, poodle, doberman, pastor alemão, pequinês e SRD) a irrigação arterial do nodo sinoatrial dependia exclu- 
sivamente do ramo distal atrial direito, o qual tem origem a partir do ramo circunflexo direito, assim como observado previamente para cães da raça doberman (Amaral 1982), pequinês (Fernandes Filho et al. 1975) e SRD (Moore 1930, James 1962, Fernandes Filho et al. 1975). Diferentemente, Biasi et al. (2013) ao examinarem corações de cães SRD mostraram que a irrigação da região tomada pelo nodo sinoatrial ficou predominantemente na dependência do ramo proximal atrial esquerdo (42,5\%), o que não coincide com a maioria dos resultados já descritos na literatura. A importância do ramo distal atrial direito, na irrigação do nodo sinoatrial dos cães, fica ainda mais evidente quando se observa que nos casos de participação de mais de um vaso no desempenho desta função, a presença deste vaso apresentou também frequência maior, ora associado ao ramo proximal atrial esquerdo, ora ao ramo proximal atrial direito. Os dados da literatura condizem com estes achados (Fernandes Filho et al. 1975, Amaral 1982).

Nesta ordem de valores entendemos que cabe destaque, em segundo lugar, ao ramo proximal atrial esquerdo, na irrigação do nodo sinoatrial, que não apenas aparece associado na maioria das vezes ao ramo distal atrial direito, como relatado anteriormente, mas também casualmente ao ramo intermédio atrial direito, assim como também demonstrado por Fernandes Filho et al. (1975) para a raça pequinês.

Os trabalhos experimentais realizados mediante ligaduras seletivas dos ramos distal atrial direito e proximal atrial esquerdo (Takayasu et al. 1962) por sua vez, também atestam a maior importância destes vasos no desempenho desta função, pois quando obstruídos ocasionam alterações consideradas tardias em decorrência de isquemia da região por eles nutridas, caracterizadas por inúmeras modificações eletrocardiográficas indicativas de extrassístoles supraventriculares, ritmo atrial ectópico, taquicardia paroxística supraventricular, marca-passo atrial mutável, onda P de voltagem, bradicardia sinusal, bloqueio sinoatrial, bem como alterações de natureza apenas imediatas e transitórias, ou seja, corrente de lesão atrial, fibrilação ventricular, distúrbio de condução intra-atrial, bloqueio A.V de primeiro grau e alterações ST/T. Todos estes fatores ressaltam a importância do assunto que também merece destaque do ponto de vista experimental (Zhang et al. 2011, Glukhov et al. 2013), uma vez que os cães são utilizados como modelos para estudos anatômicos e fisiológicos, pesquisa relacionadas à zoonoses, e para melhorar a compreensão de doenças humanas como protocolos de transplante de medula óssea e terapia genica (Schneider et al. 2008).

\section{CONCLUSÃO}

Nos cães, cabe mais frequentemente a presença do ramo distal atrial direito, ou somente ao ramo proximal atrial esquerdo, ou ainda concomitantemente a ambos, em número de vezes menor, a irrigação da área ocupada pelo nodo sinoatrial, bem como a estes vasos, o maior número de anastomoses entre as artérias que participam desta função.

Não foram identificadas, em nenhuma das raças ora examinadas, um padrão específico de irrigação do referido nodo, sendo que diferenças estatisticamente significantes de irrigação relativamente ao sexo foram apenas encontradas em cães da raça pastor alemão.
Agradecimentos.- Este trabalho representa uma compilação de resultados e interpretação de dados, frutos de pesquisas sobre a irrigação do nodo sinoatrial desenvolvidas junto ao Setor de Anatomia dos Domésticos e Silvestres da Faculdade de Medicina Veterinária e Zootecnia da Universidade de São Paulo, há mais de 3 décadas. Portanto, os autores expressam aqui a sua gratidão a todos os pesquisadores, docentes e alunos que estiveram envolvidos com o tema ao longo desses anos.

\section{REFERÊNCIAS}

Amaral C. 1982. Contribuição ao estudo da irrigação do nó sinu-atrial em cães da raça doberman. Dissertação de Mestrado, Faculdade de Medicina Veterinária e Zootecnia, Universidade de São Paulo, São Paulo. 63p.

Andretto R. 1972. Contribuição ao estudo da irrigação arterial do nó sinu-atrial, em cães da raça pastor alemão. Tese de Doutorado, Faculdade de Medicina Veterinária e Zootecnia, Universidade de São Paulo, São Paulo. 67p.

Biasi C., Borelli V., Prazeres R.F., Favaron P.O., Pavanelo Junior V., Aloia T.P.A \& Bombonato P.P. 2013. Análise comparativa entre a vascularização arterial ventricular e do nó sinoatrial em corações de cães. Pesq. Vet. Bras. 33:111-114

Fernandes Filho A., Borelli V., D’Errico A.A. \& Andretto R. 1975. Contribuição ao estudo da irrigação do sinu-atrial em cães da raça pequinês. Revta Fac. Med. Vet. Zootec. Univ. São Paulo 12:53-66.

Glukhov A.V., Hage L.T., Hansen B.J., Pedraza-Toscano A., Vargas-Pinto P., Hamlin R.L., Weiss R., Carnes C.A., Billman G.E. \& Fedorov V.V. 2013. Sinoatrial node reentry in a canine chronic left ventricular infarct model: role of intranodal fibrosis and heterogeneity of refractoriness. Circ. Arrhythm. Electrophysiol. 6:C984-94.

James T.N. 1962. Anatomy of the sinus node of the dog. Anat. Rec. 143:251265.

James T.N. 1966. Comparative ultrastructure of the sinus node in man and dog. Circulation 34:139-163.

Keith A. \& Flack M. 1907. The form and nature of the muscular connections between the primary divisions of the vertebrate heart. J. Anat. Physiol. 41:172-189.

Lobo L., Pinheiro-Vieira A., Gomes J.L., Canada N., Ribeiro L., Costa P.D., Oliveira P. \& Bussadori C. 2012. Dilated cardiomyopathy and sinoatrial dysfunction in an Estrela mountain dog. J. Am. Anim. Hosp. Assoc. 48:54-9.

Monfredi O., Dobrzynski H., Mondal T., Boyett M.R. \& Morris G.M. 2010. The anatomy and physiology of the sinoatrial node-a contemporary review. Pacing Clin. Electrophysiol. 33:1392-1406.

Moore R.A. 1930. The coronary arteries of the dog. Am. Heart J. 5:743-749.

Nomenclature I.C.V.G.A.N. 2012. Nomina Anatomica Veterinaria. Knoxville. Editorial Committee Hannover (Germany), Columbia, MO (USA), Ghent (Belgium), Sapporo (Japan). 177p.

Pereira L.P., Borelli V., Sandoli de Brito F., Fernandes Filho A., Mariano M. \& Andretto R. 1972. Enfarte atrial experimental no cão. Arch. Bras. Cardiol. 25:147-167.

Randi R.E. 1988. Contribuição ao estudo da irrigação arterial do nó sinoatrial em cães da raça boxer. Tese de Doutorado, Faculdade de Medicina Veterinária e Zootecnia, Universidade de São Paulo, São Paulo. 40p.

Rothberger C.J. \& Scherf D. 1926. Zur Kenntmis der Erregungsausbreitung von Sinusknoten auf den Vorhof. Z. Ges. Exp. Med. 53:792-835.

Schneider M.R., Wolf E., Braun J., Kolb H.J. \& Adler H. 2008. Canine embryo-derived stem cells and models for human diseases. Hum. Mol. Genet. 17:42-47.

Takayasu M., Tateishi Y., Ikuta S., Inoue Y., Kanazu I., Tamai H. \& Kato Y. 1962. An experimental study on atrial myocardial ischemia. Mie Med. J. 1:325-334.

Zhang Y., Hu D. \& Yang Z. 2013. Transplantation of pedicled autologous sinoatrial node tissue for treatment of complete atrioventricular block in dogs. Nan Fang Yi Ke Da Xue Xue Bao 33:1517-1520.

Zhang H., Lau D.H., Shlapakova I.N., Zhao X., Danilo P., Robinson R.B., Cohen I.S., Xu D.Q.Z. \& Rosen M.R. 2011. Implantation of sinoatrial node cells into canine right ventricle: biological pacing appears limited by the substrate. Cell Transplant. 20:1907-1914. 